THE PEOPLE'S MONEY 

PAOLA SUBACCHI

\section{THE PEOPLE'S \\ MONEY}

How China Is Building a Global Currency 


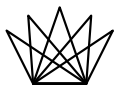

Columbia University Press

Publishers Since 1893

New York Chichester, West Sussex

cup.columbia.edu

Copyright $@ 2017$ Columbia University Press

All rights reserved

Library of Congress Cataloging-in-Publication Data

Names: Subacchi, Paola, 1962- author.

Title: The people's money : how China is building a global currency / Paola Subacchi.

Description: New York : Columbia University Press, [20I7] |

Includes bibliographical references and index.

Identifiers: LCCN 201600913I | ISBN 978023II73469 (cloth : alk. paper)

Subjects: LCSH: Foreign exchange-China. | Renminbi. | Finance-China. |

Monetary policy-China. | China-Commerce.

Classification: LCC HG3978 .S83 2016 | DDC 332.4/50951-dc23

LC record available at https://lccn.loc.gov/2016009III

Columbia University Press books are printed on permanent and durable acid-free paper.

Printed in the United States of America

c Io 98765432 I

Cover design: Mary Ann Smith

Cover image: () Getty Images 